\title{
Curvature invariants in type-III spacetimes
}

\author{
V Pravda \\ Mathematical Institute, Academy of Sciences, Žitná 25, 11567 Prague 1, Czech Republic \\ E-mail: pravda@math.cas.cz
}

Received 30 June 1999, in final form 10 August 1999

\begin{abstract}
The results of Bičák and Pravda (1998 Class. Quantum Grav. 15 1539) are generalized for vacuum type-III solutions with, in general, a non-vanishing cosmological constant $\Lambda$. It is shown that all curvature invariants containing derivatives of the Weyl tensor vanish if a type-III spacetime admits a non-expanding and non-twisting null geodesic congruence. A non-vanishing curvature invariant containing first derivatives of the Weyl tensor is found in the case of type-III spacetime with expansion or twist.
\end{abstract}

PACS numbers: 0420,0430

\section{Introduction}

In [1] we proved that in Petrov type- $\mathrm{N}$ vacuum spacetimes which admit a non-expanding and non-twisting null geodesic congruence all curvature invariants constructed from the Weyl tensor and its derivatives of arbitrary order vanish. We generalize this paper and obtain the same result for non-expanding and non-twisting Petrov type-III vacuum spacetimes. Thus it is useful to study these spacetimes in quantum gravity, as all their quantum corrections vanish (see Gibbons [2]). The proof for type-III vacuum spacetimes is based on the same ideas as that for type- $\mathrm{N}$ vacuum spacetimes given in [1]. Here we just outline the basic ideas of the proof (see section 3). For understanding and rigorous reconstruction of the proof, paper [1] is indispensable.

In the case of type-III vacuum spacetime with expansion or twist we find a non-zero curvature invariant of the first order (containing the first derivatives of the Weyl tensor).

First, let us recall some basic relations from spinor calculus and the Newman-Penrose formalism. We can use basis $o_{A}, \iota_{A}$, which satisfies

$$
o_{A} \iota^{A}=1, \quad o_{A} o^{A}=0, \quad \iota_{A} \iota^{A}=0,
$$

to decompose the Weyl spinor (see [3])

$$
\begin{gathered}
\left.\Psi_{A B C D}=\Psi_{0} \iota_{A} \iota_{B} \iota_{C} \iota_{D}-4 \Psi_{1} o_{(A} \iota_{B} \iota_{C} \iota_{D)}+6 \Psi_{2} o_{(A} o_{B} \iota_{C} \iota_{D}\right)-4 \Psi_{3} o_{(A} o_{B} o_{C} \iota_{D)} \\
+\Psi_{4} o_{A} o_{B} o_{C} o_{D}
\end{gathered}
$$


where

$$
\begin{aligned}
& \Psi_{0}=\Psi_{A B C D} o^{A} o^{B} o^{C} o^{D}, \\
& \Psi_{1}=\Psi_{A B C D} o^{A} o^{B} o^{C} \iota^{D}, \\
& \Psi_{2}=\Psi_{A B C D} o^{A} o^{B} \iota^{C} \iota^{D}, \\
& \Psi_{3}=\Psi_{A B C D} o^{A} \iota^{B} \iota^{C} \iota^{D}, \\
& \Psi_{4}=\Psi_{A B C D} \iota^{A} \iota^{B} \iota^{C} \iota^{D} .
\end{aligned}
$$

There exist four principal spinors $\alpha_{A}, \beta_{A}, \gamma_{A}, \delta_{A}$ such that

$$
\Psi_{A B C D}=\alpha_{(A} \beta_{B} \gamma_{C} \delta_{D)} .
$$

Since three principal spinors of $\Psi_{A B C D}$ coincide in type-III spacetimes, it is convenient to choose this repeated principal spinor as a basis spinor $o_{A}$. Then

$$
\Psi_{A B C D}=o_{(A} o_{B} o_{C} \delta_{D)}
$$

and

$$
\Psi_{0}=\Psi_{1}=\Psi_{2}=0 .
$$

For the Weyl spinor we thus obtain

$$
\left.\Psi_{A B C D}=-4 \Psi_{3} o_{(A} o_{B} o_{C} \iota_{D}\right)+\Psi_{4} o_{A} o_{B} o_{C} o_{D} .
$$

We choose the second basis spinor $\iota_{A}$ to satisfy

$$
D \iota_{A}=0,
$$

which implies that a complex null tetrad induced by $o_{A}$ and $\iota_{A}$ is parallelly propagated along the geodetic null congruence and several Newman-Penrose coefficients vanish:

$$
\sigma=\kappa=\varepsilon=\pi=0 .
$$

To end this section let us write down the relations

$$
\begin{aligned}
& \nabla^{A \dot{X}}=\iota^{A} \bar{\iota}^{\dot{X}} D+o^{A} \bar{o}^{\dot{X}} \Delta-\iota^{A} \bar{o}^{\dot{X}} \delta-o^{A} \bar{l}^{\dot{X}} \bar{\delta}, \\
& \nabla^{A \dot{X}} o^{B}=\gamma o^{A} o^{B} \bar{o}^{\dot{X}}-\alpha o^{A} o^{B} \bar{l}^{\dot{X}}-\tau o^{A} \iota^{B} \bar{o}^{\dot{X}}-\beta \iota^{A} o^{B} \bar{o}^{\dot{X}} \\
& +\rho o^{A} \iota^{B} \bar{\iota}^{\dot{X}}+\varepsilon \iota^{A} o^{B} \bar{\iota}^{\dot{X}}+\sigma \iota^{A} \iota^{B} \bar{o}^{\dot{X}}-\kappa \iota^{A} \iota^{B} \bar{\iota}^{\dot{X}}, \\
& \nabla^{A \dot{X}} \iota^{B}=v o^{A} o^{B} \bar{o}^{\dot{X}}-\lambda o^{A} o^{B} \bar{\iota}^{\dot{X}}-\gamma o^{A} \iota^{B} \bar{o}^{\dot{X}}-\mu \iota^{A} o^{B} \bar{o}^{\dot{X}} \\
& +\alpha o^{A} \iota^{B} \bar{\iota}^{\dot{X}}+\pi \iota^{A} o^{B} \iota^{\dot{X}}+\beta \iota^{A} \iota^{B} \bar{o}^{\dot{X}}-\varepsilon \iota^{A} \iota^{B} \bar{\iota}^{X} .
\end{aligned}
$$

Equations (1.1) and (1.7) imply that all invariant quantities constructed from $\Psi_{A B C D}$ without derivatives vanish and thus all curvature invariants of the zeroth order vanish too. In the next sections we study curvature invariants of higher orders.

\section{Expanding or twisting solutions}

Regarding (1.6) and (1.9), the Bianchi identity (see equation (7.67) in [4]) gives

$$
D \Psi_{3}=2 \rho \Psi_{3} .
$$

Using (1.1), (1.10)-(1.12), one can easily show that all first-order invariants of the Weyl tensor vanish if they contain only squares or cubes in $C_{\alpha \beta \gamma \delta ; \varepsilon}$. However, there is a non-vanishing curvature invariant

$$
I=C^{\alpha \beta \gamma \delta ; \varepsilon} C_{\alpha \mu \gamma \nu ; \varepsilon} C^{\lambda \mu \rho v ; \sigma} C_{\lambda \beta \rho \delta ; \sigma},
$$


which, in terms of Newman-Penrose quantities, reads

$$
I=\left(48 \rho \bar{\rho} \Psi_{3} \bar{\Psi}_{3}\right)^{2}
$$

The Robinson-Trautman metric of type-III, that is the general vacuum type-III solution admitting a geodesic, shear-free, twist-free and diverging null congruence, has the form

$$
\mathrm{d} s^{2}=\frac{2 r^{2}}{P^{2}} \mathrm{~d} \zeta \mathrm{d} \bar{\zeta}-2 \mathrm{~d} u \mathrm{~d} r-\left(\Delta \ln P-2 r(\ln P)_{, u}\right) \mathrm{d} u^{2}
$$

where $P(u, \zeta, \bar{\zeta})$ satisfies

$$
\Delta \Delta P=0, \quad(\Delta \ln P)_{, \zeta} \neq 0, \quad \Delta \equiv 2 P^{2} \partial_{\zeta} \partial_{\bar{\zeta}}
$$

and $\partial / \partial r$ is the repeated null eigenvector. In an appropriately chosen complex null tetrad (given, for example, in chapter 23 in [4]) we obtain

$$
\begin{aligned}
& \sigma=\kappa=\varepsilon=\pi=\Psi_{0}=\Psi_{1}=\Psi_{2}=0, \quad \rho=-\frac{1}{r}, \\
& \Psi_{3}=-\frac{P}{r^{2}}(\Delta \ln P)_{, \bar{\zeta}}, \\
& \Psi_{4}=\frac{1}{r^{2}}\left(P^{2}\left(\frac{1}{2} \Delta \ln P-r(\ln P)_{, u}\right)_{, \bar{\zeta}}\right)_{, \bar{\zeta}} .
\end{aligned}
$$

Substituting (2.6) into the invariant (2.3), we obtain

$$
I=\left(\frac{48}{r^{6}} P \bar{P}(\Delta \ln P)_{, \bar{\zeta}}(\Delta \ln \bar{P})_{, \zeta}\right)^{2} .
$$

This invariant, which is non-zero in general, can be used for analysing singularities in Robinson-Trautman solutions.

\section{Non-expanding and non-twisting solutions}

Non-expanding and non-twisting solutions satisfying (1.9) and $\rho=0$ belong to Kundt's class and they are completely known (see chapter 27.5.1. in [4]).

In [1] we have proved that for type-N vacuum spacetimes, without expansion and without twist, all curvature invariants of all orders vanish. This proof, with slight modifications, is also valid for type-III vacuum spacetimes without expansion and without twist. Thus we give here only the basic ideas of the proof.

In the following we need NP equations containing operator $D$ :

$$
\begin{aligned}
& D \tau=0, \\
& D \alpha=0, \\
& D \beta=0, \\
& D \gamma=\tau \alpha+\bar{\tau} \beta-R / 24, \\
& D \lambda=0, \\
& D \mu=R / 12, \\
& D \nu=\bar{\tau} \mu+\tau \lambda+\Psi_{3},
\end{aligned}
$$


and the commutators

$$
\begin{aligned}
& (\Delta D-D \Delta)=(\gamma+\bar{\gamma}) D-\tau \bar{\delta}-\bar{\tau} \delta, \\
& (\delta D-D \delta)=(\bar{\alpha}+\beta) D .
\end{aligned}
$$

The Bianchi identity (2.1) has the form

$$
D \Psi_{3}=0 .
$$

Let us now turn our attention to the behaviour of the NP quantities under the constant boost transformation

$$
o^{\prime A}=a o^{A}, \quad \iota^{\prime A}=a^{-1} \iota^{A} .
$$

A quantity $\Omega$, which transforms under this boost as

$$
\Omega^{\prime}=a^{q} \Omega,
$$

has the boost-weight $b(\Omega)=q$. Summary of the boost-weights for NP coefficients (NP) and operators (OP) is given in table 1 in [1]. For $\Psi_{3}$ we have

$$
\Psi_{3}^{\prime}=a^{-2} \Psi_{3} \quad \Longrightarrow \quad b\left(\Psi_{3}\right)=-2 .
$$

Now we analyse invariants of $\nabla^{C_{n} \dot{X}_{n}} \ldots \nabla^{C_{1} \dot{X}_{1}}\left(\Psi_{3} o^{(A} o^{B} O^{C} l^{D)}\right)$. The quantity $\Psi_{3} O^{(A} O^{B} O^{C} \iota^{D)}$ is invariant under the boost transformation (3.4)

$$
\Psi_{3}^{\prime} o^{\prime(A} o^{\prime B} o^{\prime C} \iota^{\prime D)}=\Psi_{3} o^{(A} o^{B} o^{C} \iota^{D)}
$$

and thus also $\nabla^{C_{n} \dot{X}_{n}} \ldots \nabla^{C_{1} \dot{X}_{1}}\left(\Psi_{3} o^{(A} o^{B} o^{C} \iota^{D)}\right)$ is invariant under (3.4) and $b\left(\nabla^{C_{n} \dot{X}_{n}} \ldots \nabla^{C_{1} \dot{X}_{1}}\right.$ $\left.\left(\Psi_{3} o^{(A} o^{B} O^{C} \iota^{D)}\right)\right)=0$. Using Leibniz's formula and relations (1.10)-(1.12), we decompose the spinor derivative $\nabla^{C_{n} \dot{X}_{n}} \ldots \nabla^{C_{1} \dot{X}_{1}}\left(\Psi_{3} o^{(A} o^{B} O^{C} \iota^{D)}\right)$ into the spinor basis of the appropriate spinor space. Each term in such a sum has the form

$$
K \underbrace{o^{A_{1}} \ldots o^{A_{m_{1}}}}_{m_{1}} \underbrace{\bar{o}^{\dot{X}_{1}} \ldots \bar{o}^{\dot{X}_{m_{2}}}}_{m_{2}} \underbrace{\iota^{B_{1}} \ldots \iota^{B_{n_{1}}}}_{n_{1}} \underbrace{l_{\dot{Y}_{1}}^{\dot{Y}_{1}} \ldots \bar{l}^{\dot{Y}_{n_{2}}}}_{n_{2}},
$$

where $K$ is a product of NP quantities. This term is also invariant under the boost (3.4) and thus

$$
b(K)=n_{1}+n_{2}-m_{1}-m_{2} .
$$

In the following we show that NP equations imply $K=0$ if $b(K) \geqslant 0$ and thus the decomposition of $\nabla^{C_{n} \dot{X}_{n}} \ldots \nabla^{C_{1} \dot{X}_{1}}\left(\Psi_{3} o^{(A} o^{B} o^{C} l^{D)}\right)$ consists only of terms containing more $o$ 's then $\iota$ 's and, as a consequence of equation (1.1), all invariants of $\nabla^{C_{n} \dot{X}_{n}} \ldots \nabla^{C_{1} \dot{X}_{1}}$ $\left(\Psi_{3} O^{(A} O^{B} O^{C} \iota^{D)}\right)$ vanish.

Lemma 1. Let an invariant constructed from the products of the spinors $\nabla^{C_{n} \dot{X}_{n}} \ldots \nabla^{C_{1} \dot{X}_{1}}$ $\left(\Psi_{3} o^{(A} o^{B} O_{C} \iota^{D)}\right)$, for fixed $n$, be non-vanishing. Then there exists a non-vanishing quantity $K=X_{1} X_{2} \ldots X_{n} \Psi_{3}, X_{i} \in N P \cup O P$ such that

$$
b\left(X_{1} X_{2} \ldots X_{n} \Psi_{3}\right)=\sum_{i=1}^{n} b\left(X_{i}\right)+b\left(\Psi_{3}\right) \geqslant 0, \quad \text { i.e. } \quad \sum_{i=1}^{n} b\left(X_{i}\right) \geqslant 2 .
$$

See the proof in [1].

We introduce a number $p$ for each NP-coefficient (or its derivatives) that describes its behaviour under the action of the operator $D$ (see [1] for the exact definition and table 2).

Comparing (3.1) and (3.2) with equations (3.2)-(3.4) in [1] we see that table 2 and lemma 2 in [1] remain unchanged. This enables us to reformulate lemma 3 (as a consequence of (3.1) (3.3)) and proposition 1. 
Lemma 3. Consider a quantity $X_{1} X_{2} \ldots X_{n}$ where $X_{i} \in N P \cup O P$.

$$
\text { If } \sum_{i=1}^{n} p\left(X_{i}\right)<0 \quad \text { then } \quad X_{1} X_{2} \ldots X_{n} \Psi_{3}=0 \text {. }
$$

The proof of lemma 3 remains unchanged.

Proposition 1. In type-III vacuum spacetimes with $\Lambda$ admitting a non-expanding and nontwisting null geodesic congruence all nth-order invariants formed from the products of spinors $\nabla^{C_{n} \dot{X}_{n}} \ldots \nabla^{C_{1} \dot{X}_{1}}\left(\Psi_{3} o^{(A} o^{B} o^{C} \iota^{D)}\right)$, with $n$ arbitrary but fixed, vanish.

Proof. We follow a similar procedure as in [1], replacing equation (3.8) in [1] by

$$
\sum_{i=1}^{n} b\left(X_{i}\right) \geqslant 2 \quad \text { and } \quad \sum_{i=1}^{n} p\left(X_{i}\right) \geqslant 0,
$$

which leads to the same conclusion.

Invariants constructed from the second term in (1.7) and its derivatives, $\nabla^{C_{n} \dot{X}_{n}} \ldots \nabla^{C_{1} \dot{X}_{1}}$ $\left(\Psi_{4} o^{A} o^{B} o^{C} o^{D}\right)$, also vanish. The proof is similar to that in [1], with the only difference

$$
\begin{aligned}
& D \Psi_{4}=0 \quad \text { for type-N, } \\
& D \Psi_{4}=(\bar{\delta}-2 \alpha) \Psi_{3}, \quad D^{2} \Psi_{4}=0 \quad \text { for type-III, }
\end{aligned}
$$

and thus we reformulate lemma 3 of [1].

Lemma 3'. Consider a quantity $X_{1} X_{2} \ldots X_{n}$ where $X_{i} \in N P \cup O P$.

$$
\text { If } \sum_{i=1}^{n} p\left(X_{i}\right)<-1 \quad \text { then } \quad X_{1} X_{2} \ldots X_{n} \Psi_{4}=0 \text {. }
$$

Proposition 1 remains unchanged.

Proposition 1'. In type-III vacuum spacetimes with $\Lambda$ admitting a non-expanding and nontwisting null geodesic congruence all nth-order invariants formed from the products of spinors $\nabla^{C_{n} \dot{X}_{n}} \ldots \nabla^{C_{1} \dot{X}_{1}}\left(\Psi_{4} O^{A} O^{B} o^{C} o^{D}\right)$, with $n$ arbitrary but fixed, vanish.

Since each term in decompositions of the spinors $\nabla^{C_{n} \dot{X}_{n}} \ldots \nabla^{C_{1} \dot{X}_{1}}\left(\Psi_{4} o^{A} o^{B} o^{C} o^{D}\right)$ and $\nabla^{C_{n} \dot{X}_{n}} \ldots \nabla^{C_{1} \dot{X}_{1}}\left(\Psi_{3} O^{(A} o^{B} o^{C} \iota^{D)}\right)$ into the spinor basis contains more $o$ 's then $\iota$ 's, we conclude our analysis with the following propositions.

Proposition 3. In type-III vacuum spacetimes with $\Lambda$, admitting a non-expanding and nontwisting null geodesic congruence, all invariants constructed from $\Psi_{A B C D}, \bar{\Psi}_{\dot{A} \dot{B} \dot{C} \dot{D}}$ and their derivatives of arbitrary order vanish.

The same proposition formulated in the tensor formalism reads:

Proposition 4. In type-III vacuum spacetimes with $\Lambda$, admitting a non-expanding and nontwisting null geodesic congruence, all invariants constructed from the Weyl tensor and its covariant derivatives of arbitrary order vanish. 


\section{Conclusion}

A general Petrov-type spacetime has non-zero curvature invariants of zeroth order. For type-N spacetimes we have shown in [1] that all Weyl invariants of all orders for non-twisting and non-expanding solutions vanish. For twisting or expanding solutions of type-N we have proven that the Weyl invariants of the zeroth and first orders vanish but we have found a non-zero invariant of the second order,

$$
C^{\alpha \beta \gamma \delta ; \varepsilon \phi} C_{\alpha \mu \gamma \nu ; \varepsilon \phi} C^{\lambda \mu \rho \nu ; \sigma \tau} C_{\lambda \beta \rho \delta ; \sigma \tau}=\left(48 \rho^{2} \bar{\rho}^{2} \Psi_{4} \bar{\Psi}_{4}\right)^{2} .
$$

In this paper we show that for type-III vacuum spacetimes without twist and expansion all Weyl invariants of all orders vanish. This fact can be used in quantum gravity (see [2]). In the case with expansion or twist only invariants of the zeroth order vanish and there exists a non-vanishing invariant of the first order,

$$
C^{\alpha \beta \gamma \delta ; \varepsilon} C_{\alpha \mu \gamma \nu ; \varepsilon} C^{\lambda \mu \rho v ; \sigma} C_{\lambda \beta \rho \delta ; \sigma}=\left(48 \rho \bar{\rho} \Psi_{3} \bar{\Psi}_{3}\right)^{2} .
$$

This invariant can be used for analysing singularities in type-III vacuum spacetimes with expansion or twist. The form of the invariant in terms of NP quantities (4.2) can also be helpful for constructing approximate solutions of Einstein's vacuum field equations in type-III with twist (see [5] for type-N).

Let us summarize our results in table 1 .

Table 1. Curvature invariants in vacuum spacetimes ( 0 , vanishes; 1 , does not vanish).

\begin{tabular}{llllll}
\hline Petrov type & I, II, D & III & III & N & N \\
\hline Expansion and twist & & $\rho \neq 0$ & $\rho=0$ & $\rho \neq 0$ & $\rho=0$ \\
Curvature invariants of order 0 & 1 & 0 & 0 & 0 & 0 \\
Curvature invariants of order 1 & 1 & 1 & 0 & 0 & 0 \\
Curvature invariants of order 2 & 1 & 1 & 0 & 1 & 0 \\
Curvature invariants of order $>2$ & 1 & 1 & 0 & 1 & 0 \\
\hline
\end{tabular}

\section{Acknowledgments}

I thank Alena Pravdová for useful discussions. I also acknowledge support from the grant GACR-201/97/0217.

\section{References}

[1] Bičák J and Pravda V 1998 Curvature invariants in type-N spacetimes Class. Quantum Grav. 151539

[2] Gibbons G W 1999 Two-loop and all-loop finite 4-metrics Class. Quantum Grav. 16 L71

[3] Penrose R and Rindler W 1984 Spinors and Spacetime 1: Two Spinor Calculus and Relativistic Fields (Cambridge: Cambridge University Press)

Penrose R and Rindler W 1986 Spinors and Spacetime 2: Spinor and Twistor Methods in Spacetime Geometry (Cambridge: Cambridge University Press)

[4] Kramer D, Stephani H, MacCallum M and Herlt E 1980 Exact Solutions of Einstein's Field Equations (Cambridge: Cambridge University Press)

[5] MacAlevey P 1999 Approximate solutions of Einstein's vacuum field equations in the type N, twisting and diverging case Class. Quantum Grav. 162259 